\title{
Collections et séries de problèmes métrologiques dans le corpus héronien et pseudo-héronien ${ }^{1}$
}

\author{
Bernard Vitrac ${ }^{\mathrm{a}}$ \\ CNRS UMR 8210 (AnHiMA), Labex HASTEC ANR-10-LABX-85, France
}

\begin{abstract}
Résumé $^{2}$. Dans cet article, nous délimitons un corpus de textes mathématiques grecs anciens selon plusieurs critères (thématique, codicologique, auctorial). La désignation n'est pas le fait des Anciens, mais la nôtre et l'un des objectifs de cet article est de justifier qu'il s'agit bien d'un corpus. Pour l'essentiel, il est composé de problèmes d'un genre particulier, les problèmes métrologiques, qui suivent un schéma formel et stylistique assez strict que nous dégageons et exemplifions. Deux autres niveaux d'analyse textuelle sont introduits : les collections - force est de constater l'écart qu'il y a parfois entre leur mode d'existence codicologique et la manière dont elles ont été éditées aux $\mathrm{XIX}^{\mathrm{e}}-\mathrm{XX}^{\mathrm{e}}$ siècles et les séries. Nous montrons que certaines de ces séries se laissent facilement identifier en tant qu'intersection de collections tandis que, pour d'autres, la cohérence est à chercher du côté d'un critère logique ou algorithmique qui correspond lui-même à un (ou plusieurs) des constituants du schéma de problème métrologique. Les particularités de ce corpus, assez différentes de celles reconnues aux compilations d'autres types de problèmes, tiennent certainement autant aux modalités de sa transmission qu'aux spécificités du domaine qu'il représente.
\end{abstract}

\begin{abstract}
Collections and series of metrological problems in the heronian and pseudoheronian corpus. In this article we define a corpus of ancient Greek mathematical texts according to several criteria: thematic, codicological, and authorial. Its designation is not ancient, but ours, and one of the aims of this article is to prove that it is indeed a corpus. It essentially consists of problems of a particular kind, namely metrological problems that fairly strictly follow a formal and stylistic scheme. We identify this scheme and give examples of it. Two other levels of textual analysis are introduced: collections and series. For the first, we discuss the gap that sometimes exists between their mode of codicological
\end{abstract}

\footnotetext{
a e-mail : bernard.vitrac [at]gmail.com

${ }^{1}$ Cette contribution a été présentée au Séminaire du programme "Séries de problèmes, un genre au carrefour des cultures », le 19 Septembre 2014. Elle a ensuite été relue par Alain Bernard et Marc Moyon. Je remercie les participants du Séminaire ainsi que mes deux relecteurs pour les remarques et suggestions qu'ils m'ont faites et qui m'ont aidé à améliorer et clarifier mon propos.

${ }^{2}$ Cet article s'appuie sur un travail sur le corpus héronien et pseudo-héronien entrepris depuis longtemps et qui est l'objet d'une longue Étude complémentaire (« La postérité des Metrica. Le corpus métrologique grec ») insérée dans l'édition (traduite et commentée) des Metrica de Héron d'Alexandrie que Fabio Acerbi et moi-même avons récemment publiée (Acerbi-Vitrac, 2014, pp. 429-588 ; les références bibliographiques complètes des ouvrages cités ici, y compris les éditions des textes héroniens, se trouvent en fin d'article). Le lecteur intéressé pourra y trouver beaucoup d'autres détails concernant Héron, les écrits qui lui sont attribués, les manuscrits, les possibles finalités, les modalités de composition et de transmission dudit corpus. Une version préliminaire de ce travail (" Héron d'Alexandrie et le corpus métrologique : état des lieux ») est disponible en ligne : http://halshs.archives-ouvertes.fr ou https://www.academia.edu/1229080/Héron_dAlexandrie_et_le_corpus_métrologique_état_des_lieux).
}

This is an Open Access article distributed under the terms of the Creative Commons Attribution License 4.0, which permits unrestricted use, distribution, and reproduction in any medium, provided the original work is properly cited. 
existence and how they were edited in the XIXth and XXth centuries. As for series, we show that some of them are easily identified as intersections of collections, while the consistency of others must be sought in a logical criterion or an algorithm. In the latter case, these criteria correspond to one (or several) of the components of the above mentioned scheme of metrological problems. The peculiarities of this corpus are quite different from those that are recognized for the compilations of other kinds of problems. They are certainly explained as much by the modalities of its transmission as by the specificities of the domain it represents.

\section{La délimitation du corpus}

Le corpus métrologique héronien et pseudo-héronien est délimité ici selon trois critères :

1) Comme son nom l'indique, il contient les collections de problèmes métrologiques attribuées à Héron $\left(\mathrm{I}^{\mathrm{er}}-\mathrm{II}^{\mathrm{e}} \mathrm{s}\right.$. de notre ère), que cette attribution soit raisonnablement indiscutable (Metrica) ou incertaine, voire totalement erronée.

2) Le second critère est l'homogénéité thématique du contenu des problèmes qui portent sur la mesure de figures géométriques, d'objets, de constructions ou sur la division des figures.

3) Le dernier est codicologique et textuel : plusieurs manuscrits indépendants contiennent à peu près les mêmes collections de problèmes, parfois avec la même attribution, souvent dans le même ordre, et on repère entre ces collections des phénomènes d'interaction textuelle (citations, portions communes).

Ce corpus ne représente évidemment pas la totalité des écrits de Héron, surtout connu pour ses ouvrages de mécanique. Son traité de la Dioptre, ouvrage qui serait pourtant pertinent quant aux mesures effectuées sur le «terrain », ne fait pas partie de ce corpus, transmis qu'il est selon une tout autre lignée textuelle. En revanche, celui des Definitiones (un recueil de définitions géométriques, partiellement attribué à notre mécanicien alexandrin par la majorité des spécialistes), accompagne les collections de problèmes.

Ce corpus ne constitue pas non plus le tout de la littérature métrologique en grec qui inclut aussi :

— des recueils de problèmes métrologiques dans des papyri ou sur des tablettes dits scolaires lesquels s'échelonnent du $\mathrm{I}^{\mathrm{er}}$ au VII ${ }^{\mathrm{e}}$ siècle ;

- le traité de Didyme d'Alexandrie (sans doute un contemporain de Héron) « sur la mesure de toutes espèces de bois » qui accompagne notre corpus, mais n'est jamais attribué à Héron.

- des tables et problèmes métrologiques, ponctuellement insérés dans d'autres types de manuscrits, notamment juridiques.

On peut leur adjoindre une littérature métrologique byzantine (Michel Psellos, Jean Pédiasimos, Isaac Argyre et quelques autres, moins connus) qui mentionne d'ailleurs souvent Héron comme autorité ou le «pille» sans dire son nom.

Il faut aussi souligner que cette délimitation et cette désignation ne sont pas le fait des Anciens, qu'elle est nôtre et donc l'un des objectifs de cet article est précisément de justifier qu'il s'agit bien d'un corpus. Reste pourtant bien des questions qui touchent aux modalités, au contexte et à l'époque de sa constitution, aux étapes successives de sa transmission et aux intentions qu'il faut prêter aux initiateurs de ces différentes phases. Outre Héron et peut-être Didyme, il semble que certains savants des écoles néo-platoniciennes de l'Antiquité tardive, plus tard les cercles érudits byzantins, actifs à Constantinople et Thessalonique, aient joué un rôle important.

Quant aux motivations, on peut imaginer la nécessité de former des «techniciens» (arpenteurs, architectes, ingénieurs) à l'Époque romaine (depuis le milieu du XIX ${ }^{\mathrm{e}}$ siècle, on tente d'établir des liens entre corpus métrologique grec et corpus agrimensorique latin ; voir Acerbi-Vitrac, 2014, pp. 519533). Plus tard, l'usage didactique de nos textes dans le cadre d'un enseignement très élémentaire de la géométrie paraît indéniable. Le souci de sauvegarde patrimoniale de textes antiques, important dans 
l'Empire byzantin pour ce qui concerne les mathématiques, a certainement joué un rôle, en particulier au moment de la copie des plus anciens témoins conservés ( $\mathrm{IX}^{\mathrm{e}}-\mathrm{X}^{\mathrm{e}}$ siècles), notamment le codex Vallae des œuvres d'Archimède (il contenait le de Mensuris attribué à Héron) et le Seragliensis gr. I.1, unique manuscrit à avoir préservé le traité des Metrica et une portion très importante du corpus (au sujet des principaux manuscrits du corpus, voir infra, ANNEXEs, 1).

\section{Le sens du terme " problème »; les problèmes métrologiques}

À l'autre extrémité du spectre en termes d'extension, on trouve le problème, entendu ici comme « unité textuelle ». Ces problèmes sont regroupés en collections (voir infra) dont la réunion constitue le corpus. D'après la vulgate historiographique, la notion de «problème » à l'œuvre dans le corpus métrologique grec relève du niveau le plus élémentaire, le plus ancien, le plus diffusé des mathématiques, censé refléter et transposer un « acte pratique » des arpenteurs, architectes et autres «ingénieurs » en vue de leur formation $^{3}$, en l'occurrence la «mesure » d'une grandeur (longueur, surface, volume) ou la « division » d'une figure (plane ou solide). Soulignons qu'il s'agit du calcul des grandeurs et non, comme cela existe dans d'autres corpus de même genre, de la mesure « réelle » avec instrument. Ajoutons encore que la dualité «mesurer / diviser » est présente dans l'ouvrage le plus ancien du corpus (le seul que l'on puisse indiscutablement attribuer à Héron), les Metrica en trois livres, livres respectivement consacrés à :

- la mesure des surfaces (planes et non planes) - livre I ;

- la mesure des volumes - livre II ;

- la division des figures planes et solides - livre III.

On retrouve cette dualité dans les traditions médiévales arabes, latines, hébraïques du mesurage, mais, dans le cas grec ancien et pour ce qui concerne le corpus métrologique, la division des figures se trouve uniquement dans le livre III des Metrica. Que celui-ci constitue une collection de problèmes, ordonnée selon différents critères : nature de la figure à diviser, nombre de portions à produire, conditions définissant le sectionnement ..., c'est évident, mais elle n'a, semble-t-il, aucunement interféré avec la portion pseudo-héronienne du corpus. Inversement, cette tradition de la division des figures est bien plus ancienne et a partie liée avec la géométrie démonstrative savante, puisque le traité le plus ancien

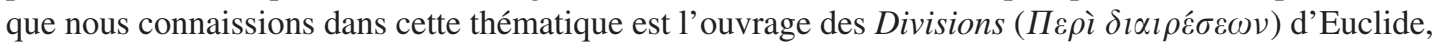
cité par Proclus et conservé partiellement dans un épitomé arabe dû à al-Sizjî. Ne disposant d'aucun terme de comparaison $\operatorname{grec}^{4}$, je limiterai donc la suite de mon propos aux problèmes métrologiques proprement dits.

Revenons à la notion de «problème » pour constater d'abord que le terme $\pi \rho o ́ \beta \lambda \eta \mu \alpha$ n'est pratiquement jamais utilisé par Héron dans ses Metrica, en particulier dans chacune des préfaces qui ouvrent ses 3 livres et qui explicitent ses intentions : son but n'est pas d'exposer des «problèmes de mesure », mais les «mesures » ( $\mu \varepsilon ́ \tau \rho \eta \sigma \varepsilon 1 \varsigma)$ elles-mêmes ! Le mot est aussi totalement absent des collections pseudo-héroniennes. On trouve « $\pi \rho \delta ́ \beta \lambda \eta \mu \alpha$ '» seulement quatre fois dans le texte des Metrica, dont trois fois dans la même formule des sections III. 12, 14, 16 : « que la droite ... soit jointe ; la droite ... sera alors celle qui réalise le problème » et une fois dans III. 18 « dès lors, que ce problème n'est pas exprimable [en nombres], c'est évident », autrement dit dans des problèmes de division que Héron traite par analyse et synthèse et qui constituent des questions d'une complexité

\footnotetext{
3 Sur cette fonction de transposition à finalité pédagogique de la tradition des collections de problèmes, voir Van Egmond, 1996, pp. 379-381 et Høyrup, 1990, 1992 et 1997.

${ }^{4}$ Cela dit, le fait que la géodésie traite aussi de la division des figures est rappelé par l'auteur de Definitiones 135.8 (HOO IV,

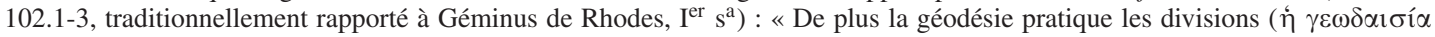

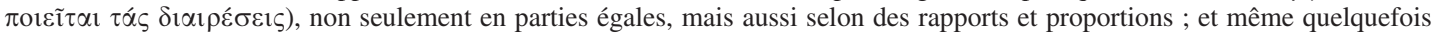
selon la qualité des terrains ».
} 


\section{SHS Web of Conferences}

supérieure à celles des deux premiers Livres. Pour le dire autrement, Héron n'est pas Diophante ${ }^{5}$ et, du moins dans les deux premiers livres de ses Metrica, il ne prétend pas enseigner à ses lecteurs la façon de résoudre des questions qui ont suffisamment d'obscurité et/ou de sophistication (selon la caractérisation du problème dialectique donnée par Aristote en Topiques I.11) pour mériter ce nom de « problème ». Les raisons qui justifient la procédure de mesure de certaines figures peuvent s'avérer difficiles, mais la question elle-même est transparente à son objet ; il n'y a là aucune énigme, aucune subtilité dans la formulation.

Bien entendu les collections de problèmes requièrent parfois de se reporter à la résolution d'un «problème» antérieur. Elles utilisent alors soit des expressions très vagues (ce qui a été écrit, ce

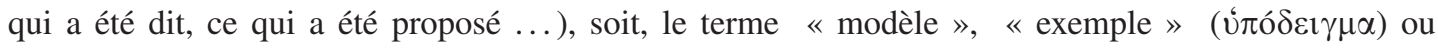
«paradigme» $(\pi \alpha \rho \alpha ́ \delta \varepsilon 1 \gamma \mu \alpha)$. Précisément exemplifions la chose, ce qui nous permettra d'introduire un premier «problème » métrologique simple, celui de la mesure des triangles équilatéraux :

« De tout triangle équilatéral, trouver l'aire.

Fais ainsi ;

Multiplie toujours l'un des côtés par lui-même et de ce à quoi s'élève le [résultat] de cette multiplication,

prends une tierce partie et un dixième.

Et c'est l'aire du triangle équilatéral.

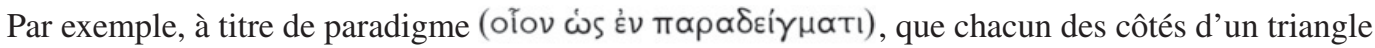
équilatéral soit de 10 cordeaux.

Trouver l'aire.

Que tu fasses ainsi ;

Les 10 d'un seul des côtés par eux-mêmes : sont produits 100 ;

Dont le $1 / 3$ : sont produits $331 / 3$;

Et le $10^{\mathrm{e}}$ : sont produits 10 ;

Ajoute $331 / 3$ et 10 : sont produits $431 / 3$;

Autant de cordeaux que cela [est] l'aire du triangle équilatéral ».

La validité de la règle est soulignée de deux manières, la mention de tout triangle équilatéral et une formulation des opérations numériques à réaliser, sans instanciation numérique. Celle-ci fait l'objet du paradigme qui suit (le triangle de côtés tous égaux à 10 cordeaux). De fait, ce genre de double énonciation est assez rare dans le corpus grec : bien plus souvent, on y trouve une succession de simples 'paradigmes' dans laquelle on fait varier les données et dont la lecture doit permettre de déterminer la procédure sous-jacente ! Voici un autre exemple :

«Une pyramide appuyée sur un carré, dont chaque côté est sur 24 pieds et l'inclinaison sur 18 pieds ;

Trouver (घúpeĩv) son volume.

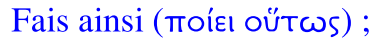

24 par eux-mêmes : sont produits (Y'́vovTaı) 576 ;

dont le $1 \frac{1}{2}$ : sont produits (YívovTaı) 288.

18 par eux-mêmes : sont produits (YívovTal) 324 ;

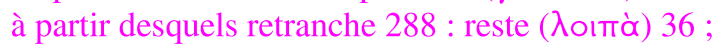

dont le côté carré produit 6 .

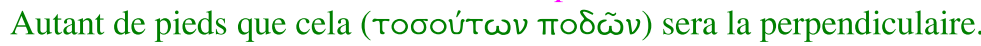

Dès lors prends le $1 / 3$ de la perpendiculaire : sont produits (Yívovtaı) 2.

Ceux-ci par les 576 : sont produits (Yívovтal) 1162.

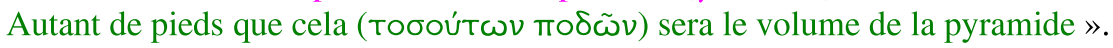

5 Voir la préface de ce dernier (DOO I, p. 2.3) et, ici même, l'article d'Alain Bernard. 
La traduction proposée ne vise pas l'élégance, mais s'emploie à respecter le caractère formulaire de la quasi totalité des problèmes métrologiques grecs anciens selon un schéma très stable indiqué ci-dessous et que le lecteur pourra facilement reconnaitre aussi dans la portion «paradigme » de la mesure des triangles équilatéraux. Particulièrement remarquables sont les traits suivants :

- la question principale est presque toujours énoncée avec une forme verbale à l'infinitif et « trouver

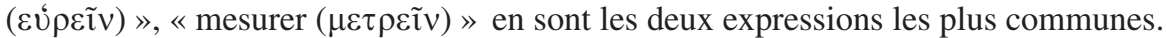

- La procédure de calcul est précédée par une phase d' "initialisation » (coloriée en bleu) à l'impératif.

- La procédure fait se succéder une alternance d'opération et de résultats partiels énoncés avec le verbe produire ( $\gamma$ í $\gamma$ vo $\mu \alpha$ l) à l'indicatif, sauf pour la soustraction (« il reste »).

- Une formule de résultat clôture ce calcul très souvent à l'aide de l'adjectif démonstratif

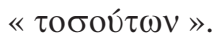

Le schéma ci-dessous (Tableau 1) constitue un idéal : il arrive que certaines formules manquent dans tel ou tel problème, sans doute l'indice d'une perte accidentelle, mais aucune des collections de problèmes de la portion pseudo-héronienne du corpus n'est systématiquement dépourvue de l'une de ces formules (question, initialisation, résultat) scandant le texte.

Il y a très peu de problèmes qui ne possèdent pas de formule de question, laquelle peut cependant être parfois sous-entendue, par exemple lorsqu'une indication de variantes de procédure ('A $\lambda \lambda \omega \varsigma$ ) fait comprendre que la question reste la même, mais doit être résolue « autrement ».

Dans le corpus métrologique grec, il y a également extrêmement peu de problèmes sans portion algorithmique, autrement dit soit des listes d'énoncés sans solution (il en existe dans les textes mathématiques babyloniens), soit des énoncés avec solution numérique, sans qu'il soit expliqué comment cette dernière a été trouvée.

Il va de soi que les désignations des parties du problème, la disposition et le coloriage ci-dessus sont de mon fait. Mais la détermination des portions successives du texte, même « copié au kilomètre » avec une mise en forme minimale dans les manuscrits, était immédiatement accessible au lecteur grâce à la régularité d'emploi de certains termes, de formes et de modes verbaux spécifiques. Même non nommées, ces parties du problème étaient identifiées par ceux qui ont composé nos collections de problèmes : nous le verrons bientôt grâce à la notion de « série ». Disons d'abord un mot sur les dites collections.

\section{Les collections du corpus héronien et pseudo-héronien}

Les Metrica de Héron forment un ouvrage divisé en trois livres, chacun consacré à un sujet bien identifié, spécifié dès la préface ; il ne contient pas seulement des problèmes métrologiques, mais aussi des théorèmes, des lemmes, des considérations métamathématiques, par exemple historiques, ainsi que la mention de divers ouvrages de la géométrie démonstrative «savante ». En principe, chaque figure est l'objet d'une section unique, la portée générale des procédures de résolution étant garantie par les démonstrations qui les accompagnent. Les problèmes métrologiques qu'on y trouve ne suivent donc pas le schéma strictement algorithmique que nous venons de mettre en évidence (Tableau 1) pour la portion pseudo-héronienne du corpus ${ }^{6}$.

Hormis ce maître-ouvrage, le corpus ne se réduit pas à un immense conglomérat de problèmes : il s'articule en collections qui, du point de vue de la transmission manuscrite, ont été traitées comme s'il s'agissait de traités. Certaines possèdent d'ailleurs un titre et une attribution. D'autres sont transmises sans titre et de manière anonyme ... Comment les définir ?

\footnotetext{
${ }^{6}$ En revanche, ce schéma s'applique aussi au traité attribué à Didyme et aux papyri dits scolaires à contenu géodésique.
} 
SHS Web of Conferences

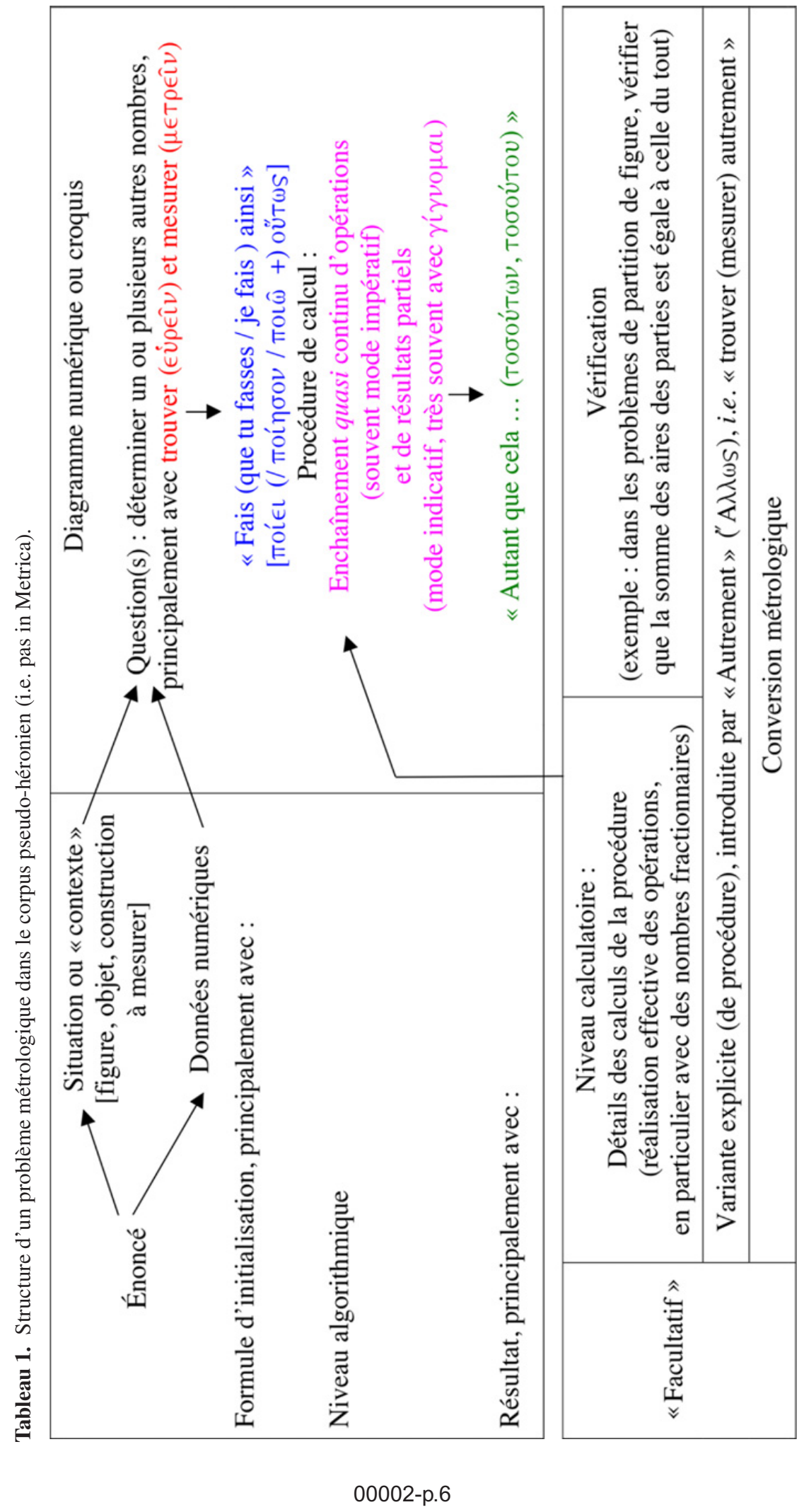


Notre seul point d'appui est constitué par les titres et les intertitres qui figurent dans les manuscrits, parfois par de simples traits ornementaux qui séparent une portion de la suivante. Plusieurs raisons font que si notre découpage (voir infra Annexes, Tableau 2a) n'est pas arbitraire, il n'est cependant pas indiscutable : le même genre d'intertitre, dans une graphie similaire, peut introduire un problème unique ou une collection (jusqu'à l'intertitre suivant) ou tout un ouvrage ; ils fluctuent dans les manuscrits, les plus récents ayant la fâcheuse tendance à être très généreux dans leurs attributions avec Héron. Certains titres n'ont manifestement pas été écrits par le copiste sur son modèle qui, peut-être, transmettait une attribution antique, mais ont été portés par une main tardive, après grattage d'un titre antérieur devenu illisible, en fonction d'une identification très spéculative ...

Malgré ces incertitudes, je crois pouvoir identifier une quinzaine de collections d'ampleur très variable et quelques problèmes «isolés ». Parmi ces collections figurent un recueil de définitions, un formulaire accompagné de tables métrologiques, le reste contenant majoritairement des problèmes. Le lecteur en trouvera la liste dans le Tableau 2a des Annexes. Sept sont (systématiquement) attribuées à Héron ( $\left.n^{\circ} 1-6+11\right)$, deux à Euclide ( $\left.{ }^{\circ} 7,9\right)$, une à Didyme ( $\left.n^{\circ} 16\right)$, une à Diophane (de Bithynie ?) ou à Diophante ( $\mathrm{n}^{\circ}$ 8). Cinq sont transmises de manière anonyme $\left(\mathrm{n}^{\circ} 10,12,13,14,15\right)$ dans le manuscrit le plus ancien (S) - son copiste attribue seulement les Metrica et une toute petite portion

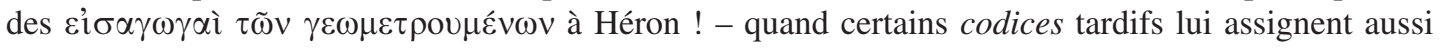
les $\mathrm{n}^{\circ}$ 12-13-14-15. Le Tableau $2 \mathrm{~b}$ indique sous quel intitulé chaque collection se retrouvent dans les éditions critiques (Hultsch, Heiberg dans $H O O$ et $M G M$ ) et donne le nombre de problèmes, plans et/ou stéréométriques, qu'elles contiennent. Le nombre total de problèmes - toujours entendu comme unités textuelles - dépasse 500, mais il y a des duplications : par exemple le problème de pyramide que nous avons cité plus haut existe en 5 exemplaires dans le corpus. Celui-ci doit contenir environ 350 problèmes véritablement distincts.

L'un des traits les plus saillants qui se dégage de cet inventaire est le contraste que l'on observe entre le statut codicologique de certaines collections composant le corpus et leurs caractéristiques textuelles. Ainsi les recueils intitulés De mensuris, Liber geeponicus, Geodaesia sont chacun transmis dans une famille de manuscrits bien identifiables, et attribués sans équivoque, ni variations, à Héron (et seulement à lui); mais le De mensuris et le Liber geeponicus sont des compilations dépourvues de toute cohérence globale, pas même celle d'un florilège : aucun auteur ne peut avoir conçu ces textes sous cette forme ${ }^{7}$. Inversement, d'autres collections possèdent une cohérence locale (sérielle !) assez forte (par exemple les $\left.N^{\circ} 6,7,10,11,15\right)$; cela dit, Geometrica, Stereometrica I et II - titres de collections éditées par Heiberg - n'ont aucune existence codicologique : il s'agit d'artefacts éditoriaux, surtout Geometrica et Stereometrica I (voir Tableau 2b des ANNEXES et Acerbi-Vitrac, 2014, pp. 462-464, 470-471).

\section{Entre problèmes et collections, les séries}

Dans son article « Types and Traditions of Mathematical Problems » (1996), Van Egmond énonçait des thèses fortes sur la constitution des séries de problèmes mathématiques : celles-ci sont des compilations d'éléments isolés et déconnectés (les problèmes), empruntés à d'autres collections soit au hasard soit pour constituer des anthologies de «favoris ». D'autre part, dans cette constitution quelque peu aléatoire des collections, les problèmes peuvent se trouver altérés : changement des paramètres ou des situations de problèmes, variation de la forme, addition d'une complication, mixage avec de nouveaux problèmes inventés pour l'occasion ..., et éventuellement réordonnés pour servir certaines intentions. Par conséquent, les outils habituels de l'analyse textuelle et de la philologie classiques sont largement sans usage pour l'étude de ces collections dont la transmission serait doublement fluide, la fluidité s'exerçant au niveau de la compilation (sélection, réorganisation) et au niveau même de la constitution

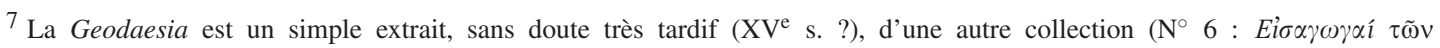
$\gamma \varepsilon \omega \mu \varepsilon \tau \rho \circ \nu \mu \varepsilon \dot{\varepsilon} \omega \nu)$.
} 
des unités textuelles (problèmes). La transmission serait donc moins le fait d'une succession de copistes que d'une multitude d' « auteurs » collaboratifs. À la traditionnelle histoire de la transmission des textes, Van Egmond propose de substituer une typologie des problèmes entendus, non plus comme des unités textuelles historiquement et linguistiquement situées, mais comme des formes mathématiques (dans les exemples qu'il donne, leur modalité d'expression est essentiellement algébrique) dont il conviendrait de suivre la circulation et les métamorphoses à travers l'histoire et les différentes civilisations.

Le corpus métrologique grec ancien permet de tempérer (ou de circonscrire) quelque peu cette forte thèse, qui contient sans doute une bonne part de vérité, mais qui n'introduit aucun niveau intermédiaire - la série ? - entre problème et collection. L'analyse de Van Egmond peut se prévaloir de la connaissance approfondie d'un vaste corpus de problèmes, mais celui-ci est, à plusieurs titres, très différent du nôtre :

— Il s'agit de « textes » beaucoup plus récents (Moyen Âge \& début de l'Époque moderne) qui n'ont pas eu à suivre le cheminement très sélectif des textes grecs anciens, notamment la translittération byzantine ;

- Ils circulent dans des milieux, par exemple les cités italiennes du Bas Moyen Âge, où la réception sociale des textes mathématiques, bientôt relayée par l'imprimerie, est incommensurable avec ce qui a existé dans l'Antiquité et même à Byzance.

- Même si, par souci de complétude et contrairement à ses prédécesseurs, Van Egmond inclut les problèmes métrologiques dans la classification des problèmes de son Appendice I (catégorie iii. 1, voir pp. 391-392 et 410), ses travaux portent avant tout sur des problèmes de caractère arithmétique dans lesquels le repérage d'ordonnancement est sans doute plus difficile à faire que dans le corpus métrologique, tributaire, au moins dans le cas grec, des classifications des lignes et des figures issues de la géométrie savante.

Lorsque l'on compare les collections composant le corpus pseudo-héronien et que l'on analyse le contenu de leurs intersections mutuelles quand celles-ci sont non vides, on constate que ces intersections (résultats d'emprunt, de compilation ... ) ne se réduisent pas à des problèmes isolés - ce cas est rare mais prennent plutôt la forme de séries, définies par un genre ou une espèce d'objets ${ }^{8}$ ou autour d'un type de questions ${ }^{9}$ [par exemple inscrire (resp. circonscrire) tel objet à (resp. dans) tel autre ... ]. C'est ce que notre schéma du Tableau 3 des AnNeXes met en évidence ${ }^{10}$.

La notion de « série » ainsi repérée peut s'analyser selon des critères non strictement textuels mais plutôt logiques, algorithmiques ou géométriques. Quant à l'extension, elle se situe à un niveau intermédiaire entre le problème individuel (unité textuelle basique) et la collection (macro-unité textuelle définie codicologiquement et philologiquement) et elle est constitutive des collections, $\mathrm{y}$ compris les florilèges ${ }^{11}$, y compris les collections apparemment les plus désordonnées (De mensuris ; Liber geeponicus). D'autres séries - il ne s'agit pas nécessairement d'intersections de collections -

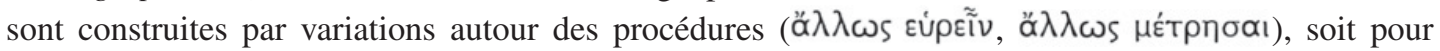
engendrer une série de problèmes, soit une série de questions à l'intérieur d'un même problème. Un cas particulier assez fréquent joue sur l'inversion du couple « donnée(s) / cherché(e)s », ce qui

\footnotetext{
${ }^{8}$ Par exemple les différentes espèces de triangles, de trapèzes, les polygones réguliers à $\mathrm{N}$ côtés $(5 \leq \mathrm{N} \leq 12)$, les différentes espèces de pyramides entières ou tronquées ...

9 Voir note 10.

${ }^{10}$ Ledit schéma cherche aussi à montrer une certaine stratification chronologique du corpus à partir de la comparaison de ces intersections de collections.

${ }^{11}$ Ainsi la portion plane de la collection $\mathrm{N}^{\circ}$ 10, dont le caractère anthologique est confirmé par la variété de ses formules de question (plus de 10 verbes différents utilisés là où des collections bien plus importantes en taille se contentent de deux ou trois) et où l'on repère une série sur le triangle rectangle, une série de "variations » sur la question «inscrire \circonscrire », une série de « variations » sur la question « mixer telles grandeurs puis les séparer »...
} 
revient à enchaîner des algorithmes inverses l'un de l'autre ${ }^{12}$. En fait, tous les éléments que nous avons identifiés comme constitutifs d'un problème métrologique : l'objet, les données, le couple « donnée(s) / cherché(e)s », la question, la procédure sont autant de prétextes à variations pour constituer des séries, ce qui confirme la pertinence de ce schéma structurel et le caractère opératoire de la notion de série.

\section{Conclusion : la spécificité des corpus métrologiques}

Cette caractéristique paraît liée autant à un mode de constitution textuel puis à un phénomène d'intertextualité dans la transmission qu'à la spécificité thématique du corpus et au statut « subordonné » (pour parler comme Aristote) de la géodésie vis-à-vis de la géométrie. Cette subordination ouvre la possibilité que les collections de problèmes représentent tout un aspect du savoir géométrique - précisément l'aspect métrologique -, par ailleurs exposé (et démontré !) dans les écrits très savants d'Eudoxe, Euclide, Archimède, Apollonius, Dionysodore... Elle est inscrite dès le début de certaines collections de problèmes qui listent les figures à traiter. Je ne suis pas sûr que l'on puisse retrouver ces caractéristiques d'ordonnancement au même degré dans les collections de problèmes arithmétiques. Même si, en l'état actuel de nos connaissances, nous n'identifions pas de processus de transmission textuelle du grec à l'arabe à la fois spécifique et massif, comme on peut en observer dans le domaine des mathématiques savantes et de l'astronomie, il est frappant de constater que plusieurs des caractéristiques du corpus métrologique grec ancien que nous avons cru pouvoir identifier ici, en particulier pour ce qui concerne l'usage des séries de problèmes, se retrouvent également dans les géométries médiévales de la mesure en Pays d’Islam et dans l'Occident latin (voir ici-même la contribution de Marc Moyon).

\section{Références bibliographiques sur Héron et le corpus pseudo-héronien}

\section{Éditions et traductions}

Codex Constantinopolitanus Palatii Veteris No. 1. E.M. Bruins (éd.), Janus Supplements volume II, 3 vol., Leiden, E. J. Brill, 1964.

Diophanti Alexandrini opera omnia cum Graeciis commentariis (DOO), edidit et latine interpretatus est P. Tannery, vol. 2, Leipzig, B. G. Teubner, 1895.

Heronis Alexandrini Geometricorum et Stereometricorum reliquiae. F. Hultsch (éd.), Berlin, Weidmann, 1864.

Heronis Alexandrini opera quae supersunt omnia (HOO). Leipzig, B. G. Teubner.

Volumen III. Rationes dimetiendi et Commentatio dioptrica, recensuit H. Schoene (1903).

Volumen IV. Heronis Definitiones cum variis collectionibus. Heronis quae feruntur Geometrica, edidit J. L. Heiberg (1912).

Volumen V. Heronis quae feruntur Stereometrica et De mensuris, edidit J. L. Heiberg (1914).

Héron d'Alexandrie, Metrica. Introduction, édition critique, traduction française et commentaires par F. Acerbi et B. Vitrac. Collection Mathematica Graeca Antiqua, 4. Pisa, Fabrizio Serra editore, 2014.

Mathematici Graeci Minores (MGM), edidit J. L. Heiberg, Det Kongelige Danske Videnskabernes Selskabs, Historisk-filologiske Meddelelser XIII,3, København, Bianco Lunos Bogtrykkeri, 1927.

P. Ver Eecke, Les opuscules mathématiques de Didyme, Diophane et Anthémius suivis du fragment mathématique de Bobbio, Paris-Bruges, Desclée, De Brouwer et Cie, 1940.

\footnotetext{
12 Quand il y a, par exemple, deux grandeurs données $\left(\mathrm{g}_{1}, \mathrm{~g}_{2}\right)$ pour en trouver une troisième $\left(\mathrm{g}_{3}\right)$, on envisagera des problèmes ou des questions en faisant toutes les permutations possibles $:\left(\mathrm{g}_{1}, \mathrm{~g}_{3}\right) \rightarrow\left(\mathrm{g}_{2}\right) ;\left(\mathrm{g}_{3}, \mathrm{~g}_{2}\right) \rightarrow\left(\mathrm{g}_{1}\right) \ldots$
} 


\section{Études}

J. Høyrup, Sub-scientific Mathematics ; Undercurrents and Missing Links in the Mathematical Technology of the Hellenistic and Roman World. Roskilde Universitetscenter, Preprints and reprints, $1990 \mathrm{n}^{\circ} 3$.

J. Høyrup, «Algèbre d'Al-gabr » et « algèbre d'arpentage » au neuvième siècle islamique et la question de l'influence babylonienne. In F. Mawet \& Ph. Talon, D'Imhotep à Copernic. Louvain, Peeters, 1992, pp. 83-110.

J. Høyrup, Hero, Ps.-Hero, and Near Eastern Practical Geometry. An Investigation of Metrica, Geometrica, and other Treatises, in K. Döring, B. Herzhoff, and G. Wöhrle (eds.), Antike Naturwissenschaft und ihre Rezeption. Band 7. Trier, Wissenschaftlicher Verlag Trier 1997, pp. 67-93.

T.-H. Martin, Recherches sur la vie et les ouvrages d'Héron d'Alexandrie, Paris, Imprimerie impériale, 1854.

W. Van Egmond, Types and Traditions of Mathematical Problems, in M. Folkerts (ed.), Mathematische Probleme im Mittelalter. Der lateinische und arabische Sprachbereich. Harrassowitz Verlag, Wiesbaden 1996, pp. 379-428.

\section{Annexes}

\section{Les manuscrits du corpus métrologique héronien et pseudo-héronien ${ }^{13}$}

Nous ne disposons d'aucun manuscrit métrologique antique grec (contrairement au cas des Agrimensores latins) : nos codices sont contemporains de - ou postérieurs à - la (première) Renaissance byzantine et à l'opération de translittération des textes $\left(\mathrm{IX}^{\mathrm{e}}-\mathrm{X}^{\mathrm{e}}\right.$ s.), i.e. en écritures minuscules. Qui pis est, dans leur très grande majorité (autour d'une centaine), ils sont tardifs $\left(\mathrm{XV}^{\mathrm{e}}-\mathrm{XVII}{ }^{\mathrm{e}} \mathrm{s}\right.$ ) et leur copie témoigne davantage de l'intérêt des humanistes pour ces textes, plutôt que d'un usage professionnel pratique. Pour la période $\mathrm{X}^{\mathrm{e}}-\mathrm{XIV}^{\mathrm{e}}$ siècle, outre les codices transmettant - de manière isolée - les compilations de compilations ou épitomés (De mensuris, Liber geeponicus, Geodaesia) ou des fragments, nous avons essentiellement 2 grands manuscrits métrologiques qui, à eux seuls, contiennent pratiquement la totalité des problèmes du corpus :

- Istanbul Seragl. gr. I.1 (S), milieu du $\mathrm{X}^{\mathrm{e}}$ s., in $\mathrm{f}^{\circ}$, parchemin, $112 \mathrm{ff}$. Copié par le moine Éphrem. Il contient une portion du corpus pseudo-héronien, le traité de Didyme et les Metrica de Héron (l'unique témoin!), donc seulement des textes antiques (au moins en théorie).

- Par. suppl. gr. 387 (C), début XIV ${ }^{\mathrm{e}}$ s., in $4^{\circ}$, papier oriental, $219 \mathrm{ff}$. Il contient des notes astronomiques et médicales, un catalogue d'étoiles, des notes chronologiques, différents manuels de calcul ( $\psi \eta \varphi \imath \varphi о \rho i ́ \alpha)$, copiés par plusieurs mains et des écrits du corpus pseudo-héronien. La portion pseudo-héronienne est d'une seule main, celle de Georgios Chumnos.

Deux ou trois autres manuscrits sont importants, car ils nous donnent accès à des états textuels différents, ou plus anciens, de certaines collections :

- Par. gr. 1670 (A), XII ${ }^{\mathrm{e}}$ s., in $4^{\circ}$, parchemin, $131 \mathrm{ff}$. Il contient des livres de comptabilité fiscale, des décrets royaux, des tables de fractions, des calculs de la date de Pâques et des écrits du corpus pseudo-héronien, à peu près les mêmes que dans $\mathbf{C}$, mais seulement en ce qui concerne les problèmes de géométrie plane.

\footnotetext{
13 Pour davantage de détails concernant les manuscrits de notre corpus, voir HOO, volume V, pp. I-CXI (en particulier XXXVII-XLV et LXVI-LXVIII) et Acerbi-Vitrac, 2014, pp. 434-445.
} 
- Par. gr. 2448 (P), début $\mathrm{XIV}^{\mathrm{e}}$ s., in $4^{\circ}$, papier, 141 ff. Il contient 2 collections métrologiques (non attribuées à Héron !) : l'opuscule dit de Diophane (ff. 70v-76v), avec le titre

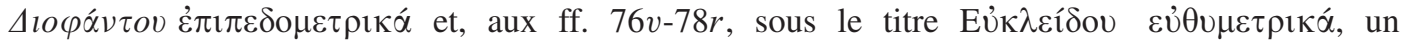
formulaire édité par Heiberg comme Geom. 22. Pour le reste, il contient les Data et la Catoptrique d'Euclide, ainsi que quelques traités de la «petite astronomie » dans des recensions propres à ce codex.

On pourrait leur adjoindre :

- Vat. gr. $215(\mathbf{V}), \mathrm{XI}^{\mathrm{e}}$ s., in $4^{\circ}$, papier oriental, $196 \mathrm{ff}$.

C'est le plus ancien témoin du (seul) Liber Geeponicus (ff. 1-24r). Le reste du codex (ff. 24r-191) contient le De re rustica (= Géoponiques !) de Cassianus Bassus le scholastique - ou plutôt la version remaniée qui en a été faite à l'époque de Constantin VII Porphyrogénète -, puis, après un folio vide, un extrait d'un cadastre de Thèbes provenant d'un autre manuscrit (ff. 193-196). 
SHS Web of Conferences

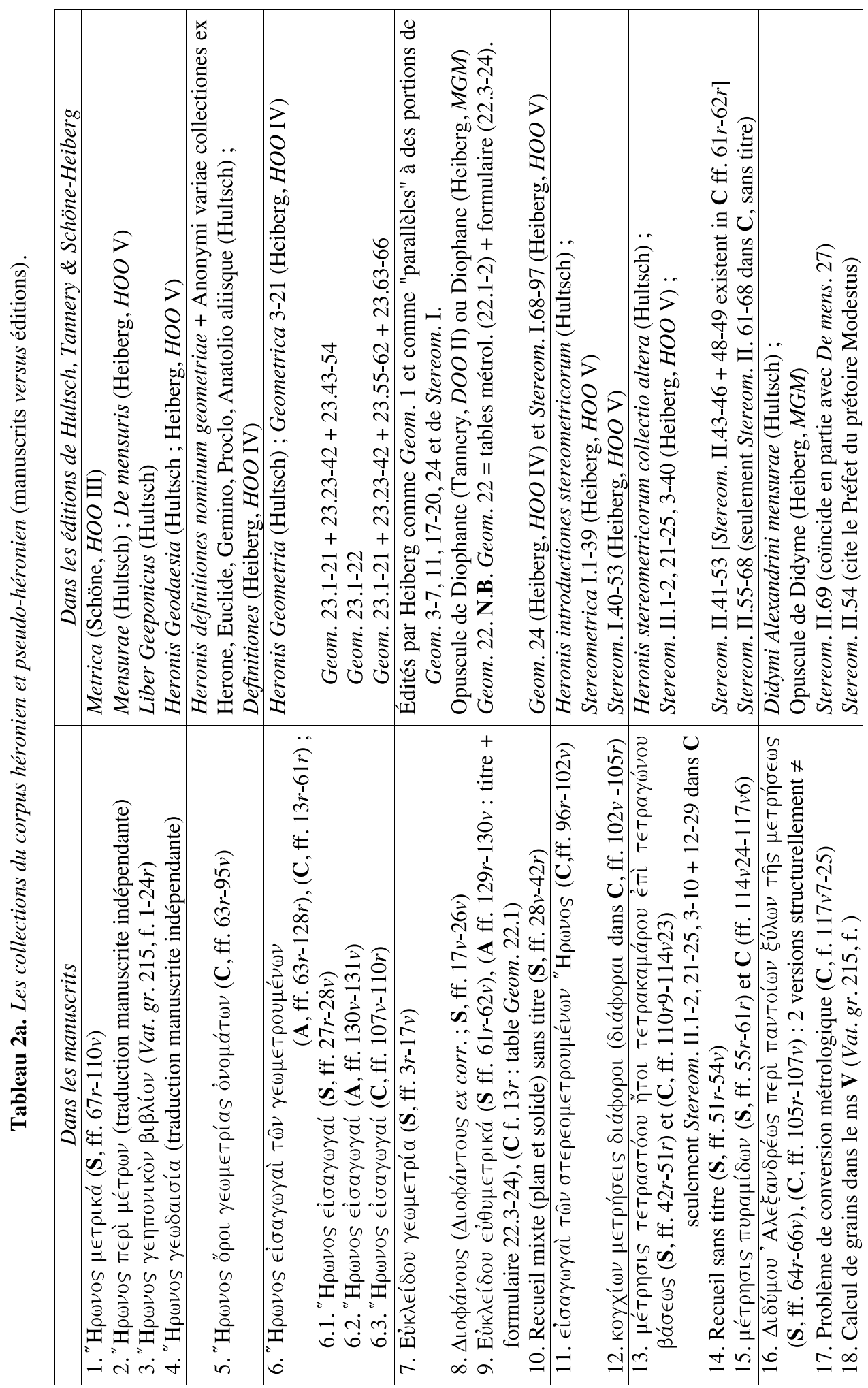


Les séries de problèmes, un genre au carrefour des cultures

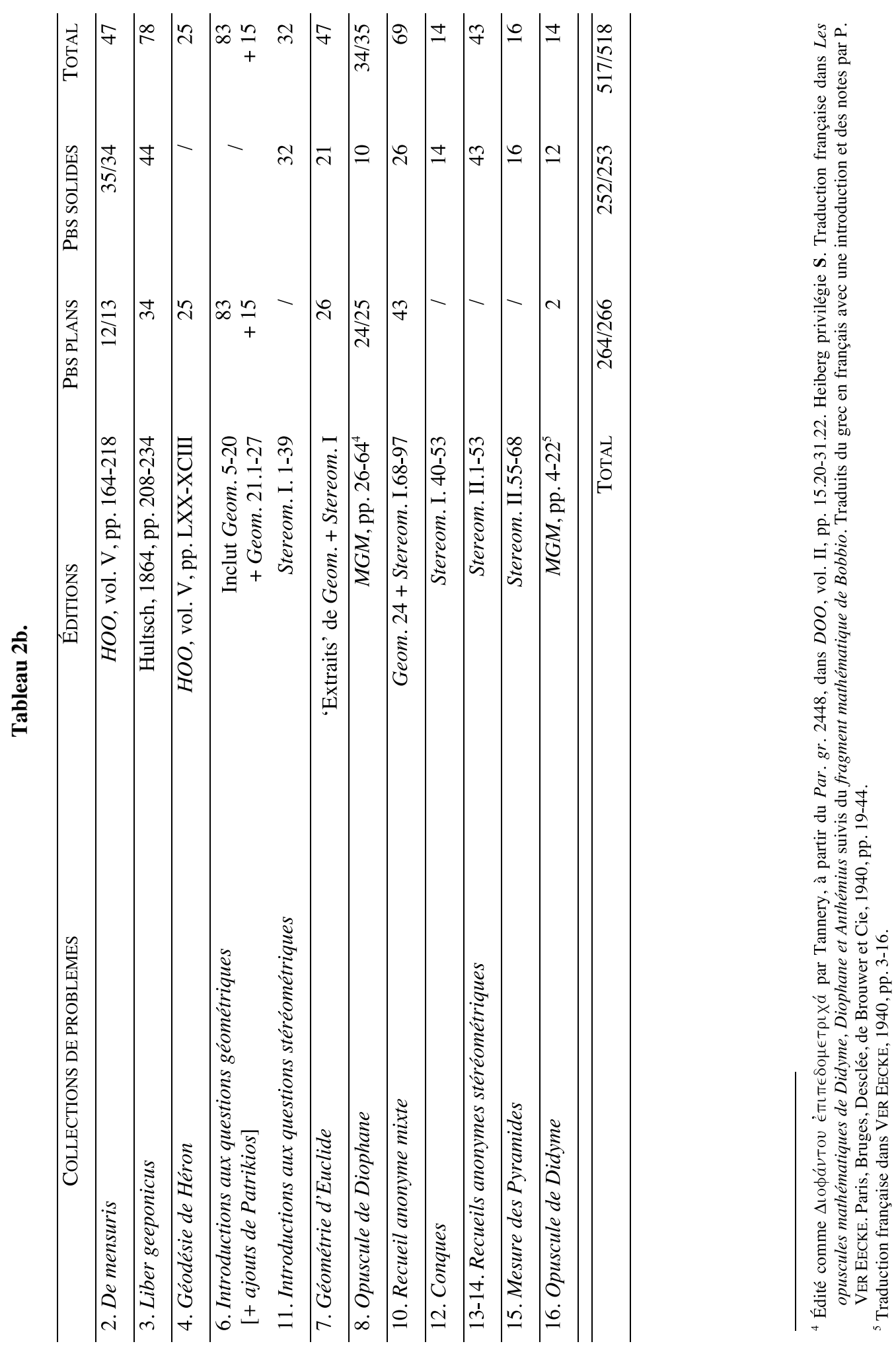




\section{Représentation du corpus métrologique pseudo-héronien basée sur l'inventaire de séries comme intersection de collections}

Le Tableau 3 infra présente les collections de problèmes (Tableau 2b) ; il ne s'agit en aucun cas d'un stemma, même si le tableau suggère une certaine sédimentation chronologique représentée « verticalement » : le haut du tableau est plus ancien que le bas.

Une flèche entre deux collections signifie qu'elles ont une intersection non vide dont le contenu est indiqué dans la «note » infrapaginale portant le numéro indiqué sur ladite flèche (de même couleur).

Une flèche unidirectionnelle indique que l'on croit pouvoir déterminer dans quel sens s'est fait l'emprunt ; dans le cas contraire, elle est à double sens. On peut certainement aller plus loin en introduisant d'autres critères, par exemple l'existence ou non de schémas et, le cas échéant, leur comparaison et, bien sûr, la confrontation philologique classique qui permet d'ordonner chronologiquement les différentes versions du texte d'un «même » problème.

Le code des couleurs (destiné à faciliter le repérage !) suggère quatre niveaux :

1. En rouge, la constitution de six collections passablement interconnectés, lesquelles ont aussi des points de contact avec les deux écrits de l'époque impériale romaine [Metrica de Héron (contacts indiscutables, sous forme de citations ; Bois et marbres de Didyme (contacts plutôt limités, voire incertains)]. Seules $(\mathbf{n})+(\mathbf{o})$ et le De mensuris semblent indépendantes et elles-mêmes interfèrent avec des collections différentes.

2. En bleu, la construction de la Géométrie dite d'Euclide ${ }^{14}$, essentiellement comme un extrait (avec variantes et quelques ajouts) des Ei $\sigma \alpha \gamma \omega \gamma \alpha i$.

3. En vert, la composition du Liber Geeponicus qui se réduit presque à la mise bout à bout d'un extrait des Definitiones, un de la Géométrie d'Euclide et un du De mensuris, avec d'importantes redites pour le dernier.

4. En gris, les constitutions de la Géodésie et des problèmes sur les conques, qui, à quelques minuscules exceptions et variantes près, reproduisent chacune une portion d'autres collections.

\footnotetext{
${ }^{14}$ La Géométrie dite d'Euclide existe (et n'existe que) dans S. Même si on fait l'hypothèse que l'extraction-compilation est contemporaine de la copie du codex - l'époque de Constantin VII Porphyrogénète -, il paraît très vraisemblable que les collections «à partir desquelles » elle a été construite [Eī $\alpha \gamma \omega \gamma \alpha i$, De mensuris, florilège (m)] ont elles-même été constituées dans l'Antiquité tardive au plus tard.
} 
Les séries de problèmes, un genre au carrefour des cultures
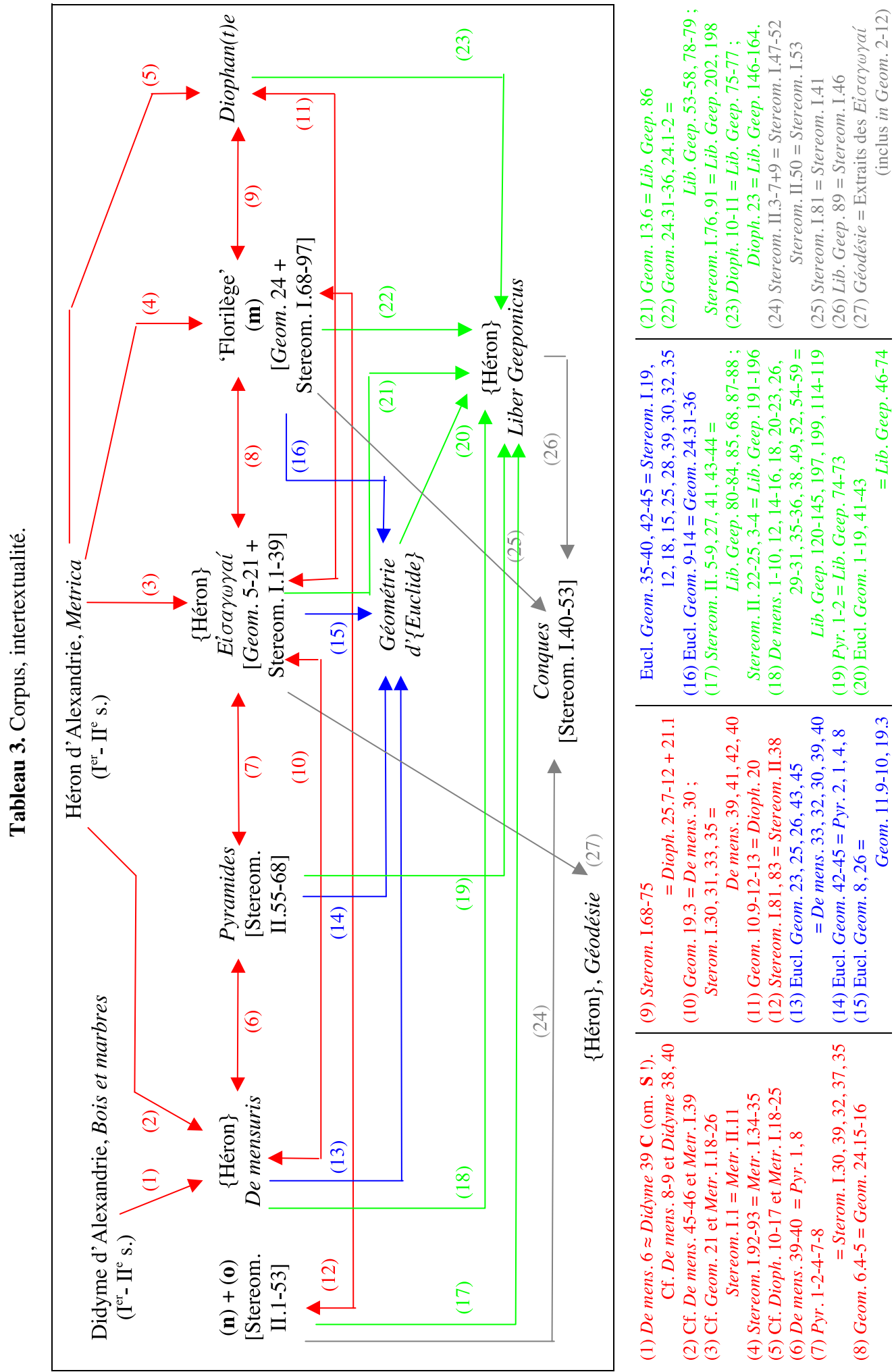